\title{
LOS DERECHOS MORALES DE AUTOR COMO DERECHOS FUNDAMENTALES EN COLOMBIA
}

DANIELA BERNAL SÁNCHEZ ${ }^{*}$ Y CARLOS CONDE GUTIÉRREZ ${ }^{* *}$

\section{INTRODUCCIÓN}

El siglo xxI, en toda su transición, ha estado inmerso en el mundo tecnológico, artístico e intelectual, que hace indiscutiblemente necesaria la implementación de protección de intangibles por medio de la propiedad industrial y los derechos de autor. En efecto, los estudios académicos en derecho y algunas otras materias, así como las diferentes industrias a nivel mundial, han sido testigos de la importancia de la propiedad intelectual en nuestros días.

Los derechos de autor, como una de las vertientes de protección de la mencionada materia, resguardan los derechos sobre las obras artísticas, las conferencias y las composiciones, por mencionar solo algunas de ellas, y asumen un papel fundamental en la producción intelectual y, en general, del tan influyente mundo de la tecnología con el que a diario tenemos contacto. Por ejemplo, el software, como el medio para ejecutar ciertas tareas por computadora, forma parte de lo que se protege por medio de los derechos de autor y que a la vez proporciona herramientas indispensables en el ámbito de las nuevas tecnologías ${ }^{1}$.

* Abogada de la Universidad Nacional de Colombia. Abogada asociada en RincónCuellar \& Asociados. Bogotá, D. C., Colombia. Correo electrónico: mdbernals@unal. edu.co. Fecha de recepción: 22 de mayo de 2017. Fecha de aceptación: 13 de octubre de 2017. Para citar el artículo: Bernal, D.; Conde, C. "Los derechos morales de autor como derechos fundamentales en Colombia", Revista La Propiedad Inmaterial n. ${ }^{\circ} 24$, Universidad Externado de Colombia, julio-diciembre 2017, pp. 53-66. Dor: https://doi. org/10.18601/16571959.n24.03

** Abogado de la Universidad Externado de Colombia. Docente e investigador de la Universidad Externado de Colombia. PhD(c) MPhil y MA, Universidad de Sheffield. Bogotá, D. C., Colombia. Correo electrónico: carlos.conde@uexternado.edu.co.

1 Para una discusión sobre el derecho de autor en el contexto de las nuevas tecnologías, ver Bernal Sánchez, Daniela y Flórez, Germán. Los nuevos modelos de distribución online en la industria de la música. En Nuevas tecnologías de la información y la comunicación, propiedad intelectual y derecho del consumo. Bogotá: Universidad Católica de Colombia, 2015; Haggart, Blayne. Copyright: The global politics of digital copyright reform. Toronto: University of Toronto Press, 2014. IsBN: 9781442614543. 
La problemática que se ha generado alrededor de estos derechos surge a partir de la protección que las distintas legislaciones, y en general los sistemas de protección intelectual, le otorgan al autor de las creaciones: el debido reconocimiento como autor, la integridad de sus obras e incluso el derecho que tiene el autor de retirar del mercado sus producciones artísticas. Esto ocurre por la división radicalizada que se les da a los dos elementos esenciales de todo derecho de autor: los derechos morales y los derechos patrimoniales; radical por cuanto adquiere una visión preponderante del aspecto económico sobre el verdadero vínculo de las obras con el autor.

Así, se puede ya concretar que por medio de los derechos patrimoniales de autor se busca la explotación económica de la producción intelectual, mientras que mediante los derechos morales de autor se pretende proteger un derecho personal referido esencialmente a la autoría de dicha producción. En cuanto a su influencia en los avances tecnológicos, artísticos y científicos, poniendo en evidencia la importancia del comercio y la internacionalización de este fenómeno, se afirma que los mismos sistemas jurídicos se encargan de darle especial protección a las prerrogativas derivadas de la explotación económica, por encima de derechos como los de integridad, modificación y retiro de obras del público, en cualquier controversia generada alrededor de derechos de propiedad intelectual ${ }^{2}$.

Para poner en evidencia esta posición, se presenta el caso de Estados Unidos, conocido como potencia mundial y que por medio de su sistema incorporado de copyright se refiere de manera preferente y especial al resguardo de derechos patrimoniales de los autores como la reproducción y distribución de las creaciones, limitando drásticamente la protección de los derechos de carácter personal solo a las "artes visuales" en términos de su integridad y atribución ${ }^{3}$.

Colombia, siendo de un sistema poco menos rígido respecto de dicha división, por medio de la Corte Constitucional como tribunal encargado de velar por la prevalencia de la Constitución Política en el ordenamiento jurídico colombiano, elevó los derechos morales de autor a la categoría de derechos fundamentales, asegurando constitucionalmente la prevalencia de estos no solo sobre los derechos patrimoniales, sino incluso frente a otras libertades y garantías constitucionales. Este fenómeno, que inició en la Corte Constitucional, se ha consolidado con dos recientes decisiones de la Dirección Nacional de Derechos de Autor (DNDA).

Por lo tanto, este artículo analiza este fenómeno y cómo estos derechos se compaginan con valores y principios constitucionales y derechos fundamentales, particularmente con la dignidad humana, en la jurisprudencia de la Corte Constitucional. En la primera parte de este escrito, se analiza el tratamiento secundario que se les da

2 Un ejemplo de esta aproximación se puede ver en Wunsch-Vincent, Sacha. The economics of copyright and the Internet. En J. M. Bauer \& M. Latzer (eds.). Handbook on the economics of the Internet. Cheltham, UK: Edward Elgar, 2016. ISBN: 9780857939852.

3 Visual Artist Right Act (VARA) 17 US Code S106. Para más información sobre VARA, ver Friedberg, Alison B. Work in progress: Reconciling vara, unfinished works, and the moral rights of artists. Tulane Journal of Technology and Intellectual Property. New Orleans: Tulane University Law School, 2010, 13, p. 217. Issn: 1533-3531. 
a los derechos morales en el copyright propio de los sistemas anglosajones, el cual se ve reflejado incluso en la tradición de Civil Law, y que se trata de la protección primaria del beneficio económico resultante de los derechos de autor. En la segunda parte, se señala la posición contraria del caso de Colombia, en donde la Corte Constitucional eleva la protección de los derechos morales de autor a derechos fundamentales; este hecho fomenta la preponderancia de los derechos morales por encima del derecho patrimonial, y se evidenciará mediante el análisis de la jurisprudencia constitucional sobre el alcance de los derechos fundamentales y el derecho moral de autor.

\section{LOS DERECHOS DE AUTOR}

Los derechos de autor son la forma de protección de propiedad intelectual en el campo literario, artístico o científico, cualquiera que sea su forma de expresión. Bajo esta circunstancia, reconocen la protección de las obras literarias, conferencias, composiciones musicales, así como de las obras artísticas, los dibujos técnicos, los mapas e incluso los documentos científicos.

Estos derechos de autor abarcan dos tipos de derechos reconocidos a nivel internacional: los derechos patrimoniales y los derechos morales. Los primeros permiten que el titular obtenga un beneficio económico por el uso de la creación por parte de terceros; los segundos protegen los intereses no patrimoniales, como el hecho de que se pueda impedir que la creación sea modificada o que determinado derecho sea reivindicado por el creador, teniendo en cuenta que la mera expresión de la obra le otorga dicha prerrogativa.

En cuanto a los derechos patrimoniales, la normativa internacional y las distintas legislaciones han entendido que estos derechos se refieren a la potestad que tiene el autor de autorizar o impedir que determinadas actividades respecto de su obra puedan ser realizadas por terceros, así como la posibilidad de remuneración económica por el uso de esta. Por esta razón, se regulan de manera particular la reproducción, la interpretación, la grabación, la radiodifusión, la traducción y la adaptación de las obras, las cuales pueden ser autorizadas por medio de contratos como el de cesión.

Sobre los derechos morales, la doctrina ${ }^{4}$ y alguna normativa internacional -como el artículo 6Bis del Convenio de Berna para la Protección de Obras Literarias y Artísticas de 1886, enmendado en 1979- determinan de manera contundente que, independientemente de los derechos patrimoniales de autor cedidos y/o autorizados por el creador, este titular conserva algunos derechos intransferibles e irrenunciables, como el de paternidad de la obra, el de oponerse a cualquier de-

4 Ver, por ejemplo, Rengifo García, Ernesto. Propiedad intelectual. El moderno derecho de autor. Bogotá: Universidad Externado de Colombia, 1997. IsBn: 9789586162777. Ver también Cornish, William; Llewelyn, David \& Aplin, Tanya. Intellectual property: Patents, copyright, trademarks and allied rights. London: Sweet \& Maxwell, 2013. ISBN: 978-0414025592. 
formación, mutilación o cualquier otra modificación, así como cualquier atentado a esta que cause perjuicio a su honor o a su reputación, y, en general, velar por la integridad de las creaciones artísticas y literarias 5 .

Las disposiciones del Convenio de Berna, así como el Tratado de la OMPI sobre Derechos de Autor de 1996 y la misma Decisión 351 de 1993 sobre el Régimen Común sobre Derechos de Autor y Derechos Conexos de la Comunidad Andina (CAN), reconocen la necesidad de proteger a los autores en el campo literario, artístico o científico, cualquiera que sea su forma de expresión. En ese sentido, han reconocido que la protección recae sobre todas las obras literarias, así como artísticas y científicas, expresadas por medio de libros, conferencias, composiciones musicales, obras dramáticas y coreográficas, ilustraciones, entre otras. Estas expresiones incluyen además los programas de ordenador, como el software.

En estricto sentido, pareciera que la protección de los dos derechos mencionados se diera en todo momento para cualquier obra protegida por los derechos de autor; sin embargo, el derecho comparado ha mostrado dos esquemas diferentes de protección que permiten hacer un cuestionamiento sobre las prerrogativas derivadas de los derechos de autor -derechos morales y derechos económicos-, por cuanto se les otorga un papel principal a los derechos patrimoniales, desconociendo la importancia de los derechos morales; estos últimos se consideran a nivel internacional como independientes de los derechos económicos, intransferibles e imprescriptibles, que se conservan incluso después de la cesión de un derecho patrimonial de autor.

Uno de los mencionados esquemas de protección corresponde al copyright, propio del Common Law, el cual, en estricto sentido, busca proteger el uso del trabajo una vez las ideas se materializan. En ese sentido, los derechos adquiridos en los países de esta corriente están directamente relacionados con la lista taxativa de derechos patrimoniales mencionada en distintas normativas internacionales, como el Convenio de Berna, otorgándole un papel secundario a los derechos morales. Esta afirmación se evidencia en la legislación de países de referencia mundial, como en Estados Unidos por medio del Copyright Act 1976 y en Inglaterra mediante el Copyright, Designs and Patents Act 1988; en el primero de estos países, por cuanto la protección de los derechos morales se brinda únicamente al trabajo de artes visuales; ${ }^{6}$ en el segundo, por cuanto la protección de dichos derechos está condicionada a la existencia y duración del reconocimiento otorgado por las autoridades compe-

5 Para más información, ver Ginsburg, Jane C. The most moral of rights: The right to be recognized as the author of one's work. Geo. Mason. J. Int'l. Commercial L. Forthcoming [Columbia Public Law Research Paper 14-517], 2016. Disponible en https:// ssrn.com/abstract=2806316 (último acceso 04.10.2017).

6 Aunque los Estados Unidos son parte del Convenio de Berna, se le cuestiona a este país por no dar una adecuada implementación al artículo $6 \mathrm{Bis}$, sobre derechos morales de autor; ver Jacobs, SAmuel. The effect of the 1886 Berne Convention on the US coyright system's treatment of moral rights and copyright term, and where that leaves US today. Michigan Telecommunications \& Technology Law Review. MI: University of Michigan Law School, 2016, 169. ISSN: 1528-8625. 
tentes del país, negando de esta forma la prerrogativa de imprescriptibilidad que afecta de manera especial el derecho de paternidad de una producción intelectual, el cual debería protegerse desde el mismo momento de creación de la obra ${ }^{7}$. Este escenario también se ve reflejado en el Acuerdo sobre los Aspectos de los Derechos de Propiedad Intelectual relacionados con el Comercio (ADPIC), de la Organización Mundial del Comercio (OMC), el cual obliga a los miembros de dicha organización a implementar de manera uniforme elementos sustanciales de la propiedad intelectual, incluyendo los derechos económicos de autor, compromiso que no se extiende a los derechos morales y cualquier otra prerrogativa que se derive de estos.

El segundo esquema de protección corresponde a la corriente de Civil Law, que, por medio de sus compendios legislativos, busca proteger de manera simultánea los derechos patrimoniales y los derechos morales, estos últimos bajo la concepción de derechos inalienables, inembargables, imprescriptibles e irrenunciables, tal como lo consigna uno de los referentes internacionales de normativa en derechos de autor como es la $\mathrm{CAN}^{8}$. Sin embargo, una vez más esta concepción pierde su relevancia y es opacada por los derechos económicos debido a la falta de conciencia de las autoridades nacionales para otorgarles una efectiva y real protección a los derechos morales de autor. Un ejemplo de esta afirmación se revela en países latinoamericanos como Colombia, en donde existen solo dos decisiones judiciales mediante las cuales se reconoce la existencia del derecho moral de autor y se toman medidas judiciales para su especial protección.

Colombia, como uno de los países que reconocen los derechos patrimoniales y morales como prerrogativas intrínsecas de todos los titulares de derechos de autor, encuentra su regulación normativa en la Decisión 351 y en la Ley 23 de 1982. Por tal circunstancia, se rige por el imperativo de que los autores tienen los derechos morales imprescriptibles e irrenunciables de conservación de la obra, reivindicación de la paternidad y posibilidad de oposición ante cualquier deformación, mutilación o modificación que atente contra el decoro o la reputación de dicho titular, así como los derechos patrimoniales para realizar, autorizar o prohibir cualquier reproducción, comunicación pública, distribución, traducción, adaptación, arreglo u otra transformación de su obra. La protección y observancia de estos derechos se llevan a cabo por medio de la Dirección Nacional de Derechos de Autor, organismo institucional delegado como autoridad administrativa y sede judicial para tales fines, y de manera especial, por la Corte Constitucional, que se ha pronunciado

7 Conde Gutiérrez, Carlos. Copyright y derechos morales de autor: la experiencia del Common Law en el Reino Unido. Revista La Propiedad Inmaterial. Bogotá: Universidad Externado de Colombia, 2011, (15), pp. 19-29. ISSN: 1657-1959. Grifftings, Jonathan. Moral rights from a copyright perspective. En Vanhees Ugent, H.; Brison, F.; Dusollier, S. \& Janssens, MC. Moral rights in the 21st Century. Belgium: Larcier, 2015. ISBN: 978-2804474751.

8 Zea Fernández, Guillermo. Derechos morales en interpretaciones o ejecuciones fijadas. La Propiedad Inmaterial. Bogotá: Universidad Externado de Colombia, 2001, (3), pp. 95-114. ISSN: 1657-1959. 
sobre la naturaleza constitucional de los derechos morales de autor y su alcance en la legislación colombiana, como se verá más adelante.

LOS DERECHOS MORALES DE AUTOR

Y LOS DERECHOS FUNDAMENTALES

La Corte Constitucional, como órgano jurisdiccional competente para adelantar el control de constitucionalidad del ordenamiento jurídico colombiano, ha elevado los derechos morales de autor a la categoría de derechos fundamentales, por cuanto protegen la "facultad creadora del hombre", inherentes a su naturaleza racional y libre, lo que le permite manifestar su espíritu por medio de su capacidad de invención y expresión de ideas o sentimientos ${ }^{9}$. Dicha categorización permite que los derechos morales de autor tengan un carácter prevalente constitucional sobre otras prerrogativas, como los mismos derechos patrimoniales de autor.

Para elevar los derechos morales a categoría fundamental ${ }^{10}$, la Corte señala que estos reúnen los requisitos esenciales de los derechos fundamentales, tales como su conexión con los principios constitucionales, contenido esencial y eficacia directa. Aunque la Corte Constitucional solo señala de manera nominal el cumplimiento de los mencionados requisitos sin adelantar un análisis detallado de cada uno de ellos en los derechos morales de autor, la misma naturaleza de estos últimos permite ver reflejados los mencionados lineamentos.

\section{LA CONEXIÓN CON LOS PRINCIPIOS CONSTITUCIONALES}

Frente a la conexión con los principios constitucionales, la Corte ha señalado que los derechos fundamentales encuentran eco directo en los principios y valores esenciales de la Constitución.

Por lo tanto, son valores constitucionales aquellos que se construyen para determinar la finalidad misma de la Constitución, el Estado y el ordenamiento jurídico. Al ser las finalidades sobre las cuales se edifican el resto de las instituciones, son expresados en forma de cláusulas generales sobre las cuales el legislador tiene competencia directa y excluyente para buscar su materialización, mientras que los jueces los deben tomar como criterios de interpretación para que así sus decisiones estén en armonía con esos valores. Estos incluyen finalidades tales como la convivencia, el trabajo, la justicia, la igualdad, el conocimiento, la libertad, la paz $^{11}$ y la dignidad humana.

A su vez, los principios constitucionales son prerrogativas generales que no obedecen a fines que se desean alcanzar, sino que, por el contrario, son máximas

9 C-155 de 1998. M.P.: Vladimiro Naranjo Mesa.

10 Para un estudio sobre los diferentes tipos de derechos fundamentales y su evolución, ver Quinche Ramírez, Manuel. Derecho constitucional colombiano de la Carta de 1991 y sus reformas. Bogotá: Universidad del Rosario, 2009. ISBN: 9789587380590.

11 T- 406 de 1992. M.P.: Ciro Angarita Pabón. 
que requieren una aplicación inmediata con el fin de delimitar cualquier conflicto o situación de disparidad que pueda afectar a los ciudadanos ${ }^{12}$. Es por medio de los principios que se establecen la organización misma del Estado y la forma como se construye el vínculo entre gobernantes y gobernados, así como las normas básicas de convivencia entre los mismos gobernados.

$\mathrm{Al}$ ser las reglas básicas sobre las que se regulan aspectos presentes, su aplicación debe estar garantizada por cualquiera de los poderes públicos: Legislativo, Ejecutivo o Judicial. Son principios constitucionales el Estado Social de Derecho, la forma de organización democrática y participativa y pluralista, la prevalencia del interés general, el trabajo, la solidaridad y el respeto por la dignidad humana ${ }^{13}$. Es sobre este último principio en el que los derechos morales de autor encuentran una conexión directa, pues, como se observa, la dignidad humana no es solo un principio, sino también un valor constitucional, e incluso se considera en sí mismo un derecho fundamental ${ }^{14}$.

En efecto, en los derechos morales la facultad creadora del hombre es inherente a su naturaleza racional y libre, los cuales son aspectos que se conectan con la dignidad humana; justamente, el principio de dignidad humana se cierne a elementos propios del ser humano, como la racionalidad ${ }^{15}$. Esta característica diferencia a los seres humanos de otros seres vivos y, a su vez, es la base que fomenta la "facultad creadora" sobre la cual se protegen los derechos morales en la jurisprudencia constitucional.

\section{CONTENIDO ESENCIAL}

El contenido o núcleo esencial es un elemento que cumple una doble función: por un lado, se establece como la parte del derecho fundamental que identifica al mismo derecho y lo hace diferente de cualquier otro tipo de derecho; por otro lado, si ese elemento determinante y diferenciador es afectado por circunstancias coyunturales -por ejemplo, los cambios en el desarrollo de políticas públicas, interpretaciones judiciales o normas contractuales-, la misma naturaleza del derecho desaparece, por lo que no se permite intervención judicial, administrativa e incluso legislativa que afecte el núcleo esencial de los derechos fundamentales ${ }^{16}$.

En efecto, es tal el celo con respecto a mantener intacta la esencia fundamental, que la misma Corte Constitucional ha establecido que ni siquiera las leyes estatutarias, entendidas como aquella normatividad consagrada constitucionalmente

14 T-881 de 2002. M.P.: Eduardo Montealegre Lynett. C-143 de 2015. M.P.: Luis Ernesto Vargas Silva.

15 T-556 de 1998. M.P.: José Gregorio Hernández.

16 T-426 de 1992. M.P.: Eduardo Cifuentes Muñoz. C-756 de 1998. M.P.: Jaime Córdoba Triviño. 
(artículo $152 \mathrm{CP}$ ) para regular aspectos relacionados con derechos fundamentales, pueden afectar el núcleo esencial ${ }^{17}$.

En relación con los derechos morales, un análisis cercano a la norma que los consagra en la legislación de derechos de autor permite precisar cuál es el núcleo esencial. La Ley 23 de 1982 claramente señala que deben entenderse como partes del derecho moral de autor la paternidad, la integridad de la obra, la conservación inédita, la modificación de la obra por parte de su autor y la facultad de retirar la obra del mercado. Cada aspecto señalado recoge la esencia misma del derecho moral de autor, la cual, como se señaló anteriormente, es la protección a la facultad creadora del hombre, inherente a su misma naturaleza.

Por lo tanto, se entiende que es el mismo legislador quien protege las prerrogativas esenciales del derecho moral de autor frente a cualquier tipo de menoscabo judicial, administrativo o contractual al señalar que estos derechos son irrenunciables, inalienables y perpetuos.

También es importante aclarar que otra función importante del núcleo esencial de los derechos fundamentales, como es el derecho moral de autor, es la protección efectiva que existe sobre este elemento, pues, como se mencionó, se desnaturaliza si se afecta su esencia fundamental. Este particular requiere un mayor análisis.

\section{EFICACIA DIRECTA}

La eficacia directa implica que la categorización de ciertos derechos como fundamentales no resulte simplemente nominal, sino que, por el contrario, al encontrarse en una jerarquía superior, su ejercicio se garantice de manera efectiva. Así pues, el ejercicio efectivo de los derechos fundamentales se puede llevar a cabo por cualquier medio que logre su adecuada protección, incluyendo la tutela, acción judicial que permite de manera sumaria y efectiva resguardar los derechos fundamentales. No obstante, a pesar de contar con una protección efectiva directa por medio de la acción de tutela, existen unos elementos mínimos que se deben considerar para proteger los derechos fundamentales por vía de tutela, los cuales provienen de la misma Constitución (artículo 86) y el desarrollo jurisprudencial de la Corte.

En primer lugar, la acción de tutela solo puede operar cuando no exista otro medio de protección frente a la presunta violación, es decir, cuando haya ausencia de mecanismos legales para su adecuada protección, lo que resalta el carácter residual o subsidiario de la tutela. En segundo lugar, es necesario que exista un inminente peligro de afectación de algún derecho fundamental, lo que requiere un mecanismo transitorio de protección hasta que dicho peligro cese o se pueda proteger por medio de otros amparos legales y judiciales. En tercer lugar, la tutela solo opera cuando existe una subordinación o indefensión, situación que ocurre, por lo general, en la relación que puede existir entre alguna autori- 
dad y un particular; este elemento resulta determinante frente a las relaciones contractuales entre particulares, pues emplear la acción de tutela para dilucidar controversia entre particulares puede afectar otros derechos fundamentales y libertades consagradas en la misma Constitución, como lo son la seguridad jurídica y el principio de la autonomía contractual ${ }^{18}$.

Lo anterior implica que la protección de los derechos fundamentales por vía de acción de tutela, si bien busca salvaguardar el núcleo esencial de cada derecho, no presupone que otros mecanismos legales que se puedan ofrecer para la protección de estos derechos sean improcedentes para proporcionar una eficacia directa. Esto es particularmente trascendental para el caso del derecho moral de autor, pues su protección efectiva se da por medio de un gran abanico legal que va desde disposiciones de tipo penal ${ }^{19}$ hasta la misma legislación de derechos de autor; aún más, esta última señala que, en el caso de que los derechos patrimoniales de autor lleguen a su fin en virtud del término del periodo de exclusividad, es el Gobierno, mediante el Ministerio de Cultura, el que debe garantizar el ejercicio efectivo de los derechos morales de paternidad e integridad de la obra ${ }^{20}$; esto claramente indica que la legislación colombiana ofrece medios efectivos de protección para el derecho moral de autor.

De igual manera, es valioso resaltar que la violación a este tipo de derechos morales ocurre, por lo general y como se observa, en la jurisprudencia de la DNDA, en relaciones contractuales entre particulares, por lo que los elementos de subsidiaridad y subordinación propios de la tutela no se encuentran presentes.

Al encontrar una conexión directa con el principio y valor constitucional de la dignidad humana, tener un contenido o núcleo esencial y gozar de eficacia directa, los derechos morales de autor pasan a una categoría superior de derechos; aún más, estos sirven como una guía interpretativa para la Corte Constitucional por cuanto, a pesar de que los derechos de autor se encuentren regulados en tratados internacionales no relacionados con derechos fundamentales, como la Decisión 351, al elevarse a la categoría de derechos fundamentales se vincula esa disposición al bloque de constitucionalidad, mecanismo que emplea tratados internacionales para hacer interpretaciones extensivas de las normas constitucionales en Colombia ${ }^{21}$.

El establecimiento de los derechos morales como categoría fundamental por parte de la Corte Constitucional indica que en Colombia se ha abierto el camino

18 T 611 de 2001. M.P.: Jaime Córdoba Triviño.

19 Ver artículo 270 del Código Penal colombiano. Ver también Osorio Moreno, César. Evolución de la protección penal del derecho de autor en Colombia. Revista de Derecho. Barranquilla: U. del Norte, 2010, (34), pp. 147-176. Issn: 0121-8697. Állvarez Álvarez, Juan Carlos; Ceballos Bedoya, María Adelaida y Muñoz Sierra, Álvaro Mauricio. De los delitos contra los derechos de autor en el Código Penal colombiano. Revista Nuevo Foro Penal. Bogotá: U. EAfIt, 2013, 9(81), 93-115. ISSN: 2539-4991. Disponible en http://publicaciones.eafit.edu.co/index.php/nuevo-foro-penal/article/view/2828 20 Artículo 30, parágrafo 3, Ley 23 de 1984.

21 C-1118 de 2005. M.P.: Clara Inés Vargas. C-1490 de 2000. M.P.: Fabio Morón. 
para ir más allá del simple reconocimiento legal en materia de propiedad intelectual para adecuarlos en un pilar del Estado: los derechos fundamentales. Esto permite otorgarles una mayor protección y prevalencia no solo frente a derechos patrimoniales de autor, sino también frente a otro tipo de libertades y garantías legales y constitucionales.

Cumpliendo con dicho propósito, con posterioridad la DNDA retomó lo dicho por la Corte al decidir sobre dos infracciones a los derechos morales de autor. En una de estas decisiones se refirió a la protección de los derechos morales de un artista, estableciendo la existencia de violación al derecho de integridad de Gabriel Antonio Calle como consecuencia de la destrucción de su mural titulado "El líder", dibujado en el centro comercial San Diego en Medellín, bajo el encargo de este último. En esta ocasión, se refirió a la vulneración del derecho fundamental como resultado de la deformación y afectación al decoro de la obra, reflejado en la eliminación total de la pureza, el recato y la estimación de esta. Frente al caso, la DNDA decidió a favor del artista y reconoció la indemnización por daños extrapatrimoniales, también hizo el reconocimiento público de los derechos de autor del artista ${ }^{22}$. De esta manera, no solo se señaló la existencia de una violación a los derechos personales del artista en relación con su obra, sino que a su vez se recalcó el rango constitucional de derecho fundamental del derecho moral de un autor.

No obstante, aun reconociendo todos los elementos fundamentales del derecho moral, el Tribunal Superior del Distrito de Bogotá - Sala Civil, en sede de apelación, estableció la seguridad pública como límite al derecho moral de autor-integridad, puesto que la obra "EL LÍDER", por encontrarse en deterioro, ponía en peligro a los transeúntes ${ }^{23}$. De igual manera, el juez de alzada encontró que el artista no demostró el ánimo de perpetuidad de la obra, pues se asumió que las actividades comerciales realizadas por el centro comercial requerían constante renovación de los espacios comunes, incluyendo el mural objeto de debate.

En un segundo momento, recurriendo nuevamente a la sentencia de constitucionalidad antes mencionada, la DNDA reconoció la violación a la prerrogativa del autor de no permitir la divulgación de la obra del fotógrafo Marcus Rodulf Loerbrok por parte del Colegio Montessori de Cartagena. Los hechos se refieren al acuerdo de pago al que llegó la mencionada institución con el señor Loerbrok, fotógrafo de profesión; el acuerdo consistía en un estudio de diez fotografías que tendría como contraprestación el pago total de la matrícula de los hijos del fotógrafo en dicha institución. Sin embargo, el colegio no reconoció la actividad como pago

22 Proceso verbal iniciado por el señor Gabriel Antonio Calle Arango contra el Centro Comercial San Diego P. H. Dirección Nacional de Derechos de Autor. Ref.: 1-2015-34057, 2016.

23 Avellaneda, Cristian [Cristian Avellaneda]. Apelación Caso CC San Diego 005 201534057 Tribunal de Bogotá [consulta: 27 noviembre de 2017] Disponible en https:// www.youtube.com/watch?v=9f5-jr13BsY\&feature=youtu.be 
y, aun sin reconocer el derecho de ineditud, realizó la publicación de las fotografías en su página web sin la autorización del autor ${ }^{24}$.

Al enarbolar los derechos morales de autor a la categoría de derechos fundamentales, Colombia va un paso más allá del reconocimiento que se le exige en tratados internacionales y regionales, como el Convenio de Berna y la Decisión 351, ofrece una garantía reforzada y los ubica de esa manera por encima de otras libertades y garantías. Dicha situación se ratifica por medio de las dos recientes decisiones de la DNDA mencionadas, las cuales encuentran su base en la protección constitucional otorgada por la Corte Constitucional.

\section{CONCLUSIONES}

Debido a diferentes factores económicos, sociales y políticos, los derechos de autor se han sumergido en la preponderancia del factor económico por encima del reconocimiento personal de los autores de las creaciones intelectuales. En ese sentido, a nivel internacional se ha garantizado de manera prevalente y casi que exclusiva la protección de los derechos patrimoniales de autor, hasta tal punto que incluso las disputas y las consecuentes decisiones judiciales se toman con base en la infracción de derechos de carácter patrimonial, que en nada se relacionan con el derecho de integridad o modificación de los contenidos, por mencionar solo algunos de los derechos morales de autor.

Esto se ve reflejado en escenarios de protección de talla mundial, como el compendio normativo de ADPIC, que excluye el amparo del derecho moral al establecer que, en virtud del acuerdo, ningún miembro tiene derecho ni obligación respecto de los derechos conferidos por el artículo 6Bis del Convenio de Berna, ni de los derechos que se derivan de este. En igual medida, los países que son parte del sistema de protección de corte anglosajón copyright, mediante sus distintas regulaciones nacionales, se enfocan en la protección de los derechos morales cuando exista un reconocimiento del derecho patrimonial por parte de las autoridades competentes; dicha protección se centra de manera exclusiva en la distribución y/o reproducción autorizada de las obras.

Evidentemente, desde las normas que propenden hacia la estandarización de los derechos de propiedad intelectual, se excluyen los derechos morales de autor, los cuales tienen relación directa con el principio de la dignidad humana, como así lo ha reconocido la máxima autoridad constitucional colombiana por medio de sus sentencias de constitucionalidad. Dicho principio constituye uno 
de los pilares sobre los cuales se soportan muchos de los derechos que no pueden ser limitados por los Estados.

En cuanto a la corriente de Civil Law, es necesario mencionar que la protección de los derechos morales resulta limitarse al espectro formal por su mención en los cuerpos normativos, y no a la materialización de estos. Así las cosas, a pesar de la inclusión de los derechos morales de autor en las regulaciones nacionales, regionales e internacionales, como el caso del Convenio de Berna, los países se han quedado cortos en cuanto al reconocimiento efectivo de dichos derechos, y ello se ve reflejado en el número de procedimientos administrativos y decisiones judiciales enfocados en dicho propósito.

Colombia, en su intento de proteger los derechos morales de autor, le ha otorgado una potestad indudablemente certera a la Corte Constitucional, que les ha dado un valor primario y no secular a estos derechos. Por medio de su sentencia de constitucionalidad, señaló que el derecho moral de autor reúne los tres elementos propios de los derechos fundamentales (conexión directa con los principios y valores constitucionales, núcleo esencial y eficacia directa), de esa manera enarboló el contenido fundamental del derecho moral de autor, mencionando que la facultad creadora es parte de la existencia, la racionalidad y, por tanto, de la dignidad del ser humano; este último principio es uno de aquellos por los que indistintamente los países han otorgado valor de carácter fundamental a muchos de los derechos inviolables del hombre.

De la misma forma sustentada en la decisión de la máxima autoridad constitucional del país, la DNDA ha dado pequeños pasos que propenden hacia la protección de los derechos morales por medio de dos sentencias mediante las cuales decide salvaguardar, por un lado, la integridad de una obra, y por el otro, el derecho a la ineditud de una producción intelectual, que se refiere al derecho del autor de que su obra no sea publicada bajo ninguna circunstancia.

Por todo lo anterior, se evidencia cómo Colombia ha ido un paso más allá de lo que muchos países han llegado en su propósito de proteger los derechos morales de autor. En efecto, mediante el reconocimiento formal y material de estos derechos, hasta el punto de poner en evidencia su carácter fundamental, Colombia ha mostrado la importancia de tales derechos por encima del beneficio económico-patrimonial que pueda resultar de, por mencionar solo un ejemplo, la reproducción autorizada de una producción intelectual.

\section{BIBLIOGRAFÍA}

JURISPRUDENCIA

\section{Constitutional}

C-155 de 1998. M.P.: Vladimiro Naranjo Mesa. 
C 756 de 1998. M.P.: Jaime Córdoba Triviño.

C-1490 de 2000. M.P.: Fabio Morón.

C-143 de 2015. M.P.: Luis Ernesto Vargas Silva.

C-1118 de 2005. M.P.: Clara Inés Vargas.

C-993 de 2004. M.P.: Jaime Araújo Rentería.

T- 406 de 1992. M.P.: Ciro Angarita Pabón.

T-426 de 1992. M.P.: Eduardo Cifuentes Muñoz.

T-556 de 1998. M.P.: José Gregorio Hernández.

T 611 de 2001. M.P.: Jaime Córdoba Triviño.

T-881 de 2002. M.P.: Eduardo Montealegre Lynett.

\section{Dirección Nacional de Derechos de Autor}

Proceso verbal iniciado por el señor Gabriel Antonio Calle Arango contra el Centro Comercial San Diego P. H. Dirección Nacional de Derechos de Autor. Ref.: 1-2015-34057, 2016.

Proceso verbal iniciado por el señor Marcus Ingo Rudolf Loerbroks contra el Colegio Montessori Limitada. Dirección Nacional de Derechos de Autor. Ref.: 1-2015-63182.

\section{Doctrina}

Álvarez Álvarez, Juan Carlos; Ceballos Bedoya, María Adelaida y Muñoz Sierra, Álvvaro Mauricio. De los delitos contra los derechos de autor en el Código Penal colombiano. Revista Nuevo Foro Penal. Bogotá: U. eafit, 2013, 9(81), 93-115. ISSN: 2539-4991. Disponible en <http://publicaciones.eafit. edu.co/index.php/nuevo-foro-penal/article/view/2828>

Bernal Sánchez, Daniela y Flórez, Germán. Los nuevos modelos de distribución online en la industria de la música. Nuevas tecnologias de la información y la comunicación, propiedad intelectual y derecho del consumo. Bogotá: Universidad Católica de Colombia, 2015.

Conde Gutiérrez, Carlos. Copyright y derechos morales de autor: la experiencia del Common Law en el Reino Unido. Revista La Propiedad Inmaterial. Bogotá: Universidad Externado de Colombia, 2011, (15), pp. 19-29. Issn: 1657-1959.

Cornish, William; Llewelyn, David \& Aplin, Tanya. Intellectual property: Patents, copyright, trademarks and allied rights. London: Sweet \& Maxwell, 2013. ISBN: 978-0414025592.

Ginsburg, Jane C. The most moral of rights: The right to be recognized as the author of one's work. Geo. Mason. J. Int'l. Commercial L. Forthcoming [Columbia Public Law Research Paper 14-517], 2016. Disponible en https://ssrn. com/abstract $=2806316$ (último acceso 04.10.2017) 
Grifftings, Jonathan. Moral rights from a copyright perspective. En Vanhees Ugent, H.; Brison, F.; Dusollier, S. \& Janssens, Mc. Moral rights in the 21st Century. Belgium: Larcier, 2015. IsBn: 978-2804474751.

Friedberg, Alison B. Work in progress: Reconciling vara, unfinished works, and the moral rights of artists. Tulane Journal of Technology and Intellectual Property. New Orleans: Tulane University Law School, 2010, 13. IssN: 1533-3531.

Haggart, BlaYne. Copyright: The global politics of digital copyright reform. Toronto: University of Toronto Press, 2014. IsBN: 9781442614543.

Jacobs, Samuel. The effect of the 1886 Berne Convention on the US coyright system's treatment of moral rights and copyright term, and where that leaves US today. Michigan Telecommunications \& Technology Law Review. MI: University of Michigan Law School, 2016, 169. IsSN: 1528-8625.

Osorio Moreno, César. Evolución de la protección penal del derecho de autor en Colombia. Revista de Derecho. Barranquilla: U. del Norte, 2010, (34), pp. 147-176. ISSN: 0121-8697.

Quinche Ramírez, Manuel. Derecho constitucional colombiano de la Carta de 1991 y sus reformas. Bogotá: Universidad del Rosario, 2009. IsBN: 9789587380590.

Rengifo García, Ernesto. Propiedad intelectual. El moderno derecho de autor. Bogotá: Universidad Externado de Colombia, 1997. IsBn: 9789586162777. Triana Uribe, Tatiana Andrea. Por desconocer el derecho moral del fotógrafo Marcus Igno Rudolf Loerbroks, la Dirección Nacional de Derecho de Autor condenó al Colegio Montessori Limitada de Cartagena. Revista La Propiedad Inmaterial. Bogotá: Universidad Externado de Colombia, 2017, (23), 279285. ISSN: 1657-1959.

Wunsch-Vincent, Sacha. The economics of copyright and the Internet. In J. M. Bauer \& M. Latzer (eds.). Handbook on the economics of the Internet. Cheltham, UK: Edward Elgar, 2016. IsBN: 9780857939852.

Zea Fernández, Guillermo. Derechos morales en interpretaciones o ejecuciones fijadas. La Propiedad Inmaterial. Bogotá: Universidad Externado de Colombia, 2001, (3), pp. 95-114. ISSN: 1657-1959. 